\title{
Hölder Estimates for Nonlocal-Diffusion Equations with Drifts
}

\author{
Zhen-Qing Chen • Xicheng Zhang
}

Received: 30 October 2014 / Accepted: 23 December 2014 / Published online: 15 February 2015

(C) School of Mathematical Sciences, University of Science and Technology of China and Springer-Verlag Berlin Heidelberg 2015

\begin{abstract}
We study a class of nonlocal-diffusion equations with drifts, and derive a priori $\Phi$-Hölder estimate for the solutions by using a purely probabilistic argument, where $\Phi$ is an intrinsic scaling function for the equation.
\end{abstract}

Keywords Parabolic function - Hölder regularity - Nonlocal operator - Drift • Space-time Hunt process · Lévy system

Mathematics Subject Classification Primary 60H30 - 35K05 .

Secondary $47 \mathrm{G} 20 \cdot 60 \mathrm{~J} 45$

\section{Introduction}

It is well known that a priori Hölder estimates play a key role in the study of nonlinear equations. There is a wealth literature on this for differential operators. In this paper, we study a priori Hölder estimates of parabolic functions for a large class of timedependent nonlocal operators with (time-dependent) gradient drifts on $\mathbb{R}^{d}$

$$
\mathscr{L}_{t}^{b} u=\mathscr{L}_{t} u+b_{t} \cdot \nabla u
$$

\footnotetext{
Z.-Q. Chen $(\varangle)$

Department of Mathematics, University of Washington, Seattle, WA 98195, USA

e-mail: zqchen@uw.edu

X. Zhang

School of Mathematics and Statistics, Wuhan University, Wuhan 430072, Hubei,

People's Republic of China

e-mail: XichengZhang@gmail.com
} 
Here $b_{t}(x)$ is an $\mathbb{R}^{d}$-valued measurable function on $[0, \infty) \times \mathbb{R}^{d}$ and $\mathscr{L}_{t}$ is a timedependent purely nonlocal operator given by

$$
\mathscr{L}_{t} u(x)=\int_{\mathbb{R}^{d}}[u(x+z)+u(x-z)-2 u(x)] \kappa_{t}(x, z) \mathrm{d} z,
$$

where $\kappa_{t}(x, z)$ is symmetric in $z$ (i.e., $\left.\kappa_{t}(x, z)=\kappa_{t}(x,-z)\right)$ and satisfies

$$
\sup _{t, x} \int_{\mathbb{R}^{d}}\left(1 \wedge|z|^{2}\right) \kappa_{t}(x, z) \mathrm{d} z \leqslant C_{1}
$$

and for some regularly varying function $\phi$ with index $\alpha \in[0,2]$ (see Definition 3.1 below),

$$
\frac{c_{1}}{\phi(|z|)|z|^{d}} \leqslant \kappa_{t}(x, z) \leqslant \frac{c_{1}^{-1}}{\phi(|z|)|z|^{d}} \text { for }|z| \leqslant 3 .
$$

Note that under condition (1.2), $\mathscr{L}_{t} u(x)$ is well defined and bounded for every $u \in$ $C_{b}^{2}\left(\mathbb{R}^{d}\right)$. Since $\kappa_{t}(x, z)$ is symmetric in $z$, we can rewrite $\mathscr{L}_{t} u(x)$ in (1.1) as

$$
\begin{aligned}
\mathscr{L}_{t} u(x) & =2 \mathrm{p} . \mathrm{v} \cdot \int_{\mathbb{R}^{d}}(u(x+z)-u(x)) \kappa_{t}(x, z) \mathrm{d} z \\
& =2 \int_{\mathbb{R}^{d}}\left(u(x+z)-u(x)-\nabla u(x) \cdot z \mathbf{1}_{|z| \leqslant 1}\right) \kappa_{t}(x, z) \mathrm{d} z
\end{aligned}
$$

for $u \in C_{b}^{2}\left(\mathbb{R}^{d}\right)$.

When $\kappa_{t}(x, z)=c|z|^{-d-\alpha}$ for some suitable constant $c>0$ and $\alpha \in(0,2), \mathscr{L}_{t}$ is just the usual fractional Laplacian $\Delta^{\alpha / 2}$. Recently Silvestre [13] proved the following a priori Hölder estimate for the fractional-diffusion equation with drift

$$
\partial_{t} u=\Delta^{\alpha / 2} u+b_{t} \cdot \nabla u \text {. }
$$

There are constants $C>0$ and $\beta \in(0,1)$ such that for any classical solution $u$ of Eq. (1.4), $x, y \in \mathbb{R}^{d}$ with $|x-y| \leqslant 1$ and $0<s \leqslant t \leqslant 1$,

$$
|u(t, x)-u(s, y)| \leqslant C\|u\|_{L^{\infty}\left([0,1] \times \mathbb{R}^{d}\right)} \frac{|x-y|^{\beta}+|t-s|^{\beta / \alpha}}{t^{\beta / \alpha}},
$$

provided $b$ in the Hölder class $C^{1-\alpha}$ when $\alpha \in(0,1)$, and bounded measurable when $\alpha \in[1,2)$, where the constants $C$ and $\beta$ only depend on $d, \alpha,\|b\|_{\infty}$, as well as on the $(1-\alpha)$-Hölder norm of $b$ when $\alpha \in(0,1)$. See [9] for recent result on local regularity of solutions to

$$
\Delta^{\alpha / 2} u+b(x) \cdot \nabla u=f
$$

in Sobolev spaces with $\alpha \in(0,1)$. 
In the literature, if $\alpha \in(1,2)$, the Eq. (1.4) is usually referred to as the subcritical case since the fractional Laplacian $\Delta^{\alpha / 2}$ is of higher order than that of the gradient part $b_{t} \cdot \nabla$. For $\alpha=1$, it is called the critical case since the fractional Laplacian has the same order as the first order gradient term. For $\alpha \in(0,1)$, it is known as the supercritical case because the fractional Laplacian is of lower order than the drift term, and the drift term can be stronger than the diffusion term in small scales. This explains why one needs $b$ to be Hölder continuous for the above a priori estimate in the supercritical case. It should be noted that the following scaling property plays a crucial role in paper [13]: for $\lambda>0$, let $u^{\lambda}(t, x):=\lambda^{-\alpha} u\left(\lambda^{\alpha} t, \lambda x\right)$ and $b^{\lambda}(t, x):=b\left(\lambda^{\alpha} t, \lambda x\right)$, then $u^{\lambda}$ satisfies

$$
\partial_{t} u^{\lambda}=\Delta^{\alpha / 2} u^{\lambda}+\lambda^{\alpha-1} b^{\lambda} \cdot \nabla u^{\lambda}
$$

We mention that the Hölder estimate (1.5) has been used in the study of well-posedness of multidimensional critical Burger's equations in Zhang [14].

In this paper, we are concerned with the following nonlocal-diffusion equation with drift $b$ :

$$
\partial_{t} u=\mathscr{L}_{t}^{b} u=\mathscr{L}_{t} u+b_{t} \cdot \nabla u
$$

Following [11], we define

$$
\Phi(r):=\left(\int_{r}^{2} \frac{\mathrm{d} s}{s \phi(s)}\right)^{-1}, \quad r \in(0,1)
$$

which is a continuous increasing function. The purpose of introducing this function $\Phi$ is to deal with the case when $\phi$ in (1.3) is a regularly varying function of order 0 . It is known (see (3.5) below) that $\lim _{r \rightarrow 0} \frac{\Phi(r)}{\phi(r)}=\alpha$. Thus $\phi$ and $\Phi$ are comparable when $\alpha \in(0,2]$ so we could use $\phi$ in place of $\Phi$ in this case. The function $\Phi$ will be used to measure the modulus of continuity of parabolic functions; see Theorem 1.1. Such a result would be trivial if $\lim _{r \rightarrow 0} \Phi(r)>0$. Thus without loss of generality, we will assume in this paper that

$$
\lim _{r \rightarrow 0} \Phi(r)=0
$$

Note that

$$
\lim _{r \rightarrow 0} \Phi(r)=0 \Longleftrightarrow \int_{0}^{2} \frac{\mathrm{d} s}{s \phi(s)}=\infty \stackrel{(1.3)}{\Longleftrightarrow} \int_{\mathbb{R}^{d}} \kappa_{t}(x, z) \mathrm{d} z=\infty .
$$

In [11], Kassmann and Mimica called $\Phi$ an intrinsic scaling function and obtained $\Phi$ Hölder regularity of harmonic functions for the (time-independent) nonlocal operator $\mathscr{L}_{0}$ with $b_{0}=0$ under conditions (1.2), (1.3) and (1.8). When $\kappa_{0}(x, z)$ satisfies (1.3) for all $z \in \mathbb{R}^{d}$ with $\phi(r)=r^{\alpha}$ for some $0<\alpha<2$ and $b_{0}=0$, Hölder regularity of harmonic functions of $\mathscr{L}_{0}$ was first established in Bass and Levin [2]. For a priori Hölder estimate for symmetric jump processes, see, e.g., [5, 6].

In this paper, we use ":=" as a way of definition. Let $\mathbb{R}^{+}:=[0, \infty)$, and $C_{c}^{\infty}\left(\mathbb{R}^{+} \times\right.$ $\mathbb{R}^{d}$ ) the space of smooth functions with compact support in $\mathbb{R}^{+} \times \mathbb{R}^{d}$. A probability 
measure $\mathbf{Q}$ on the Skorokhod space $\mathbb{D}\left([0, \infty) ; \mathbb{R}^{+} \times \mathbb{R}^{d}\right)$ is said to be a solution to the martingale problem for $\left(\mathscr{L}_{t}^{b}, C_{c}^{\infty}\left(\mathbb{R}^{+} \times \mathbb{R}^{d}\right)\right)$ with initial value $(t, x) \in \mathbb{R}^{+} \times \mathbb{R}^{d}$ if $\mathbf{Q}\left(Z_{0}=(t, x)\right)=1$ and for every $f \in C_{c}^{\infty}\left(\mathbb{R}^{+} \times \mathbb{R}^{d}\right)$,

$$
M_{s}^{f}:=f\left(s+t, X_{s}\right)-f\left(t, X_{0}\right)-\int_{0}^{s}\left(\partial_{r}+\mathscr{L}_{t+r}^{b}\right) f\left(t+r, X_{r}\right) \mathrm{d} r
$$

is a $\mathbf{Q}$-martingale. The martingale problem for $\left(\mathscr{L}_{t}^{b}, C_{c}^{\infty}\left(\mathbb{R}^{+} \times \mathbb{R}^{d}\right)\right)$ with initial value $(t, x) \in \mathbb{R}^{d}$ is said to be well posed if it has a unique solution.

Throughout this paper, we assume conditions (1.2) and (1.3) and (1.8) hold. Here are our main results.

Theorem 1.1 Assume that the martingale problem for $\left(\mathscr{L}_{t}^{b}, C_{c}^{\infty}\left(\mathbb{R}^{+} \times \mathbb{R}^{d}\right)\right)$ is well posed for every initial value $(t, x) \in \mathbb{R}^{+} \times \mathbb{R}^{d}$. Let $\Phi$ be defined by (1.7).

(i) Suppose that $\liminf _{r \rightarrow 0} r / \Phi(r)=0$ and for some $C_{2}>0$,

$$
\frac{\Phi(r)}{\Phi(s)} \leqslant C_{2} \frac{r}{s} \text { for } 0<s \leqslant r \leqslant 1
$$

and $b_{t}(x)$ is continuous in $x$ for each $t>0$ having

$$
\|b /(1+|x|)\|_{\infty}:=\sup _{t \in[0,1], x \in \mathbb{R}^{d}}\left|b_{t}(x)\right| /(1+|x|)<\infty
$$

with

$$
\left|b_{t}(x)-b_{t}(y)\right| \leqslant C_{b}|x-y| / \Phi(|x-y|) \text { for }|x-y| \leqslant 1
$$

for some $C_{b}>0$. Then there are constants $\beta \in(0,1)$ and $\lambda=\lambda(\| b /(1+$ $\left.|x|) \|_{\infty}\right)>0$ such that for any classical solution $u$ of (1.6), and for any $0<t \leqslant$ $t_{0} \leqslant 1$ and $\left|x_{0}-x\right|+\lambda\left(t_{0}-t\right) \leqslant \Phi^{-1}\left(t_{0}\right)$,

$\left|u\left(t_{0}, x_{0}\right)-u(t, x)\right| \leqslant 16\|u\|_{L^{\infty}\left([0,1] \times \mathbb{R}^{d}\right)} t_{0}^{-\beta}\left(\left(t_{0}-t\right)+\Phi\left(\left|x_{0}-x\right|+\lambda\left(t_{0}-t\right)\right)\right)^{\beta}$.

(ii) Suppose $\liminf _{r \rightarrow 0} r / \Phi(r)>0$ and b is a bounded measurable function on $\mathbb{R}^{+} \times \mathbb{R}^{d}$. Then there is a constant $\beta \in(0,1)$ such that for any classical solution $u$ of (1.6), and for any $0<t \leqslant t_{0} \leqslant 1$ and $\left|x_{0}-x\right| \leqslant \Phi^{-1}\left(t_{0}\right)$,

$$
\left|u\left(t_{0}, x_{0}\right)-u(t, x)\right| \leqslant 16\|u\|_{L^{\infty}\left([0,1] \times \mathbb{R}^{d}\right)} t_{0}^{-\beta}\left(\left(t_{0}-t\right)+\Phi\left(\left|x_{0}-x\right|\right)\right)^{\beta} .
$$

Remark 1.2 Condition (1.10) is automatically satisfied by (3.1) below when $\phi$ (also $\Phi)$ is regularly varying with $\alpha \in[0,1)$. Moreover, condition (1.10) is satisfied when $\Phi$ is comparable to a convex function $\Psi$ with $\Psi(0)=0$ as in this case $r / \Psi(r)$ is 
increasing. The following table gives some examples of regularly varying functions $\phi$ that satisfy conditions (1.2), (1.3) and (1.8), where the $\alpha$ stands for the index of regularly varying function $\phi$, and the last column denotes the modulus of continuity in spatial variable $x$ required for $\mathbb{R}^{d}$-valued function $b_{t}(x)$.

\begin{tabular}{lllll}
\hline Case & $\alpha$ & $\phi(s)$ & $\Phi(r)$ & $b$ \\
\hline (i) & 0 & $\ln \frac{3}{s}$ & $\asymp\left(\ln \ln \frac{3}{r}\right)^{-1}$ & $s \ln \ln \frac{3}{s}$ \\
(i) & 0 & 1 & $\left(\ln \frac{2}{r}\right)^{-1}$ & $s \ln \frac{2}{s}$ \\
(i) & 0 & $\left(\ln \frac{3}{s}\right)^{-1}$ & $\asymp\left(\ln \frac{3}{r}\right)^{-2}$ & $s\left(\ln \frac{3}{s}\right)^{2}$ \\
(i) & $(0,1)$ & $s^{\alpha}$ & $\asymp r^{\alpha}$ & $s^{1-\alpha}$ \\
(i) & 1 & $s\left(\ln \frac{3}{s}\right)^{\beta}, \beta>0$ & $\asymp r\left(\ln \frac{3}{r}\right)^{\beta}$ & $\left(\ln \frac{3}{s}\right)^{-\beta}$ \\
(ii) & 1 & $s\left(\ln \frac{3}{s}\right)^{\beta}, \beta \leqslant 0$ & $\asymp r\left(\ln \frac{3}{r}\right)^{\beta}$ & 1 \\
(ii) & $(1,2)$ & $s^{\alpha}$ & $\asymp r^{\alpha}$ & 1 \\
(ii) & 2 & $s^{2}\left(\ln \frac{3}{s}\right)^{\beta}, \beta>1$ & $\asymp r^{2}\left(\ln \frac{3}{r}\right)^{\beta}$ & 1 \\
\hline
\end{tabular}

Remark 1.3 When $\phi(r)=r^{\alpha}$ with $\alpha \in(0,2)$ (and so $\Phi(r)$ is comparable to $r^{\alpha}$ ), condition $\liminf _{r \rightarrow 0} r / \Phi(r)=0$ corresponds precisely to the supercritical case $0<$ $\alpha<1$, while $\liminf _{r \rightarrow 0} r / \Phi(r)>0$ corresponds to $1 \leqslant \alpha<2$. So Theorem 1.1 contains the main results of Silvestre [13] as a particular case. Unlike [13], in the supercritical case (i), we do not need to assume $b$ is bounded. Moreover, in this paper we can not only deal with more general but also time-dependent nonlocal operator $\mathscr{L}_{t}^{b}$. In particular, taking $b=0$, we obtain a priori $\Phi$-Hölder estimates for parabolic functions of time-dependent nonlocal operator $\mathscr{L}_{t}$. It contains as a special case a priori $\Phi$-Hölder estimates for harmonic functions $u(x)$ of nonlocal operator $\mathscr{L}_{0}$, which is the main result of Kassmann and Mimica [11] when $b_{0}=0$.

Remark 1.4 In this paper, we concentrate on a priori Hölder estimates for parabolic functions. For results on the well-posedness of the martingale problems for $\mathscr{L}_{t}^{b}$, we refer the reader to $[1,7,8]$ and the references therein. We remark here that if $\kappa_{t}(x, z)$ is independent of $(t, x)$, then $\mathscr{L}_{t}=\mathscr{L}_{0}$ is the generator of a Lévy process $Y$. When $b_{t}(x)$ is uniformly Lipschitz in $x$, it is easy to show that for every initial data $(t, x) \in \mathbb{R}^{+} \times \mathbb{R}^{d}$, stochastic differential equation

$$
\mathrm{d} X_{s}^{t}=\mathrm{d} Y_{s}+b_{t+s}\left(X_{s}^{t}\right) \mathrm{d} s
$$

has a unique strong solution $X^{t}$ with $X_{0}^{t}=x$. (This can be done as follows. For each $\omega \in \Omega, \operatorname{ODE} d Z_{s}^{t}=b_{t+s}\left(Z_{s}^{t}+Y_{s}(\omega)\right) \mathrm{d} s$ with $Z_{0}^{t}(\omega)=x$ has a unique solution. Then $X_{s}^{t}:=Z_{s}^{t}+Y_{t}$ is the unique solution to $\operatorname{SDE}(1.14)$ with $X_{0}^{t}=x$.) Hence in this case, the martingale problem for $\left(\mathscr{L}, C_{c}^{\infty}\left(\mathbb{R}^{+} \times \mathbb{R}^{d}\right)\right)$ is well posed and so Theorem 1.1 is applicable. It is important to note that the $\Phi$-Hölder estimate in Theorem 1.1 does not depend on the Lipschitz constant of $b_{t}(x)$.

When the martingale problem for $\left(\mathscr{L}_{t}^{b}, C_{c}^{\infty}\left(\mathbb{R}^{+} \times \mathbb{R}^{d}\right)\right)$ is well posed for every initial value $(t, x) \in \mathbb{R}^{+} \times \mathbb{R}^{d}$, there is a space-time Hunt process $Z_{s}=\left(V_{0}+s, X_{s}\right)$ 
having $\partial_{s}+\mathscr{L}_{V_{0}+s}^{v}$ as its infinitesimal generator. For any bounded classical solution $u$ of (1.6), by Itô's formula, $u\left(s+t, X_{S}\right)$ is a $\mathbf{P}_{(t, x)}$-martingale for every $(t, x) \in \mathbb{R}^{+} \times \mathbb{R}^{d}$. This is the only property of $u$ we used in the proof of Theorem 1.1. Hence the conclusion of Theorem 1.1 holds for any bounded function $u$ on $\mathbb{R}^{+} \times \mathbb{R}^{d}$ such that $u\left(s+t, X_{s}\right)$ is a $\mathbf{P}_{(t, x)}$-martingale for every $(t, x) \in \mathbb{R}^{+} \times \mathbb{R}^{d}$.

The approach of this paper is purely probabilistic. Our tool is the timeinhomogeneous strong Markov process $X$ determined by the solution of the martingale problem for $\left(\mathscr{L}_{t}^{b}, C_{c}^{\infty}\left(\mathbb{R}^{+} \times \mathbb{R}^{d}\right)\right)$. In Sect. 2, we prove an abstract result on Hölder's continuity in terms of certain estimates on exiting and hitting probabilities, which is motivated by the approaches in $[2,5]$. This probabilistic approach has its origin in Krylov and Safanov [12] for diffusion processes associated with second order nondivergence form differential operators. In Sect. 3, we prove our main results by verifying the abstract conditions. The Lévy system of the strong Markov process $X$ plays a key role in establishing these exiting and hitting probabilities.

\section{An Abstract Criterion for Hölder's Regularity}

Let $\Omega$ be the space of càdlàg functions from $\mathbb{R}^{+}=[0, \infty)$ to $\mathbb{R}^{d}$, which is endowed with the Skorokhod topology. Let $X_{s}(\omega)=\omega_{s}$ be the coordinate process over $\Omega$. Define the space-time process

$$
Z_{s}:=\left(V_{s}, X_{s}\right), \quad V_{s}:=V_{0}+s .
$$

Let $\left\{\mathscr{F}_{s}^{0} ; s \geqslant 0\right\}$ be the natural filtration generated by $X$. Suppose $\left\{\mathbb{P}_{(t, x)} ; t \geqslant\right.$ $\left.0, x \in \mathbb{R}^{d}\right\}$ is a family of probability measures over $\left(\Omega, \mathscr{F}_{\infty}^{0}\right)$ so that $Z=$ $\left(\Omega, \mathscr{F}_{\infty}^{0}, \mathscr{F}_{s}^{0}, Z_{s}, \mathbb{P}_{(t, x)}\right)$ is a time-homogenous strong Markov processes with state space $\mathbb{R}^{+} \times \mathbb{R}^{d}$ with

$$
\mathbb{P}_{(t, x)}\left(Z_{0}=(t, x)\right)=1
$$

Denote by $\left\{\mathscr{F}_{s}: s \geqslant 0\right\}$ the minimal augmented filtration of $Z$. Note that under $\mathbb{P}_{(t, x)}$, $\left\{X_{s}^{t}:=X_{s+t} ; s \geqslant 0\right\}$ is a possibly time-inhomogeneous strong Markov process with

$$
\mathbb{P}_{(t, x)}\left(X_{s}^{t}=x, s \in[0, t]\right)=1 .
$$

For a Borel set $A \subset \mathbb{R}^{+} \times \mathbb{R}^{d}$, denote by $\sigma_{A}, \tau_{A}$ the hitting time and exit time of $A$, i.e.,

$$
\sigma_{A}:=\inf \left\{s \geqslant 0: Z_{s} \in A\right\}, \quad \tau_{A}:=\inf \left\{s \geqslant 0: Z_{s} \notin A\right\}
$$

Definition 2.1 A nonnegative Borel measurable function $u(t, x)$ on $\mathbb{R}^{+} \times \mathbb{R}^{d}$ is called $Z$-harmonic (or simply parabolic) in a relatively open subset $D$ of $\mathbb{R}^{+} \times \mathbb{R}^{d}$ if for each relatively compact open subset $A \subset D$ and every $(t, x) \in A$,

$$
u(t, x)=\mathbb{E}_{(t, x)}\left[u\left(Z_{\tau_{A}}\right)\right] .
$$


Remark 2.2 Condition (2.1) is equivalent to the following. For any $\left(\mathscr{F}_{S}\right)$-stopping time $\tau$,

$$
u(t, x)=\mathbb{E}_{(t, x)}\left[u\left(Z_{\tau \wedge \tau_{A}}\right)\right]
$$

Indeed, assume that (2.1) holds for any $(t, x) \in A$. In view of $Z_{\tau} \in A$ on $\left\{\tau<\tau_{A}\right\}$, we have

$$
\mathbf{1}_{\tau<\tau_{A}} u\left(Z_{\tau}\right)=\mathbf{1}_{\tau<\tau_{A}} \mathbb{E}_{Z_{\tau}}\left[u\left(Z_{\tau_{A}}\right)\right]
$$

Let $\left\{\theta_{t} ; t \geqslant 0\right\}$ be the usual shift operators on $\Omega$. By the strong Markov property, we have

$$
\begin{aligned}
\mathbb{E}_{(t, x)}\left[\mathbf{1}_{\tau<\tau_{A}} u\left(Z_{\tau}\right)\right] & =\mathbb{E}_{(t, x)}\left[\mathbf{1}_{\tau<\tau_{A}} \mathbb{E}_{Z_{\tau}}\left[u\left(Z_{\tau_{A}}\right)\right]\right] \\
& =\mathbb{E}_{(t, x)}\left[\mathbf{1}_{\tau<\tau_{A}} \mathbb{E}_{(t, x)}\left[u\left(Z_{\tau_{A}} \circ \theta_{\tau}\right) \mid \mathscr{F}_{\tau}\right]\right] \\
& =\mathbb{E}_{(t, x)}\left[\mathbf{1}_{\tau<\tau_{A}} u\left(Z_{\tau_{A}} \circ \theta_{\tau}\right)\right] \text { since }\left\{\tau<\tau_{A}\right\} \in \mathscr{F}_{\tau} .
\end{aligned}
$$

Since $\tau_{A}=\tau+\tau_{A} \circ \theta_{\tau}$ on $\left\{\tau<\tau_{A}\right\}$ and $Z_{\tau_{A}} \circ \theta_{\tau}=Z_{\tau+\tau_{A} \circ \theta_{\tau}}$, we obtain

$$
\mathbb{E}_{(t, x)}\left[\mathbf{1}_{\tau<\tau_{A}} u\left(Z_{\tau}\right)\right]=\mathbb{E}_{(t, x)}\left[\mathbf{1}_{\tau<\tau_{A}} u\left(Z_{\tau_{A}}\right)\right]
$$

which implies that

$$
\begin{aligned}
\mathbb{E}_{(t, x)}\left[u\left(Z_{\tau \wedge \tau_{A}}\right)\right] & =\mathbb{E}_{(t, x)}\left[\mathbf{1}_{\tau<\tau_{A}} u\left(Z_{\tau}\right)\right]+\mathbb{E}_{(t, x)}\left[\mathbf{1}_{\left\{\tau \geqslant \tau_{A}\right\}} u\left(Z_{\tau_{A}}\right)\right] \\
& =\mathbb{E}_{(t, x)}\left[u\left(Z_{\tau_{A}}\right)\right]=u(t, x) .
\end{aligned}
$$

Let $\Phi:(0,2) \rightarrow[0, \infty)$ be a continuous and strictly increasing function with $\Phi(1)=1$. Write for $r>0$,

$$
B(r):=\left\{z \in \mathbb{R}^{d}:|z|<r\right\} \text { and } Q(r):=[0, \Phi(r)) \times B(r) .
$$

Define

$$
\varphi_{a}(r):=\Phi^{-1}(a \Phi(r)) \text { for } a>0, \quad D_{a}(r):=Q\left(\varphi_{a}(r)\right) \backslash Q\left(\varphi_{\sqrt{a}}(r)\right) \text { for } a>1
$$

Notice that

$$
\varphi_{1}(r)=r \quad \text { and } \quad a \mapsto \varphi_{a}(r) \text { is strictly increasing. }
$$

We make the following assumptions: 
$\left(\mathbf{H}_{1}\right)$ There exist constants $C_{3}, C_{4} \geqslant 1$ such that for each $a \geqslant C_{4}$ and $r, R \in(0,1)$ with $\varphi_{a}(r) \leqslant R$,

$$
\sup _{\left(t_{0}, x_{0}\right) \in Q(r)} \mathbb{P}_{\left(t_{0}, x_{0}\right)}\left(X_{\tau_{Q(r)}} \notin B(R)\right) \leqslant C_{3} \frac{\Phi(r)}{\Phi(R)} .
$$

$\left(\mathbf{H}_{2}\right)$ There is an increasing sequence of positive numbers $\left\{a_{k} ; k \geqslant 1\right\} \subset(1, \infty)$ with $\lim _{k \rightarrow \infty} a_{k}=\infty$ such that for every $a \in\left\{a_{k} ; k \geqslant 1\right\}$, there exists a constant $\gamma_{a} \in(0, a]$ so that for each $r \in(0,1)$ with $\varphi_{a}(r) \leqslant 1$, there is a radon measure $\mu_{r}$ over $D_{a}(r)$ such that for any compact subset $K \subset D_{a}(r)$ with $\mu_{r}(K) \geqslant \frac{1}{3} \mu_{r}\left(D_{a}(r)\right)$,

$$
\inf _{\left(t_{0}, x_{0}\right) \in Q(r)} \mathbb{P}_{\left(t_{0}, x_{0}\right)}\left(\sigma_{K}<\tau_{Q\left(\varphi_{a}(r)\right)}\right) \geqslant \frac{\gamma_{a}}{a},
$$

and $\lim _{k \rightarrow \infty} \gamma_{a_{k}}=\infty$.

Remark 2.3 If $X_{s}$ is a continuous process, then $\left(\mathbf{H}_{1}\right)$ is automatically satisfied.

Theorem 2.4 Under $\left(\mathbf{H}_{1}\right)$ and $\left(\mathbf{H}_{2}\right)$, there exists a constant $\beta \in(0,1)$, which only depends on $C_{3}, C_{4}$, and $\gamma_{a}$, such that for each $r \in(0,1)$, every bounded measurable function $u$ on $[0,1] \times \mathbb{R}^{d}$ that is parabolic in $Q(r)$,

$$
|u(t, x)-u(0,0)| \leqslant 8\left(\frac{t \vee \Phi(|x|)}{\Phi(r)}\right)^{\beta}\|u\|_{L^{\infty}\left([0, \Phi(r)] \times \mathbb{R}^{d}\right)} \quad \text { for }(t, x) \in Q(r) \text {. }
$$

Proof Our proof is adapted from Chen and Kumagai [5, Theorem 4.14]. Fix $r \in(0,1)$. Without loss of generality, we may assume $0 \leqslant u \leqslant 1$ on $[0, \Phi(r)] \times \mathbb{R}^{d}$. Otherwise, instead of $u$, we may consider

$$
\tilde{u}_{t}(x)=\frac{u_{t}(x)-\inf _{(s, y) \in[0, \Phi(r)] \times \mathbb{R}^{d} u_{s}(y)}}{\sup _{(s, y) \in[0, \Phi(r)] \times \mathbb{R}^{d}} u_{S}(y)-\inf _{(s, y) \in[0, \Phi(r)] \times \mathbb{R}^{d}} u_{S}(y)} .
$$

(i) Define for $n \in \mathbb{N}$,

$$
r_{n}:=\varphi_{a^{1-n}}(r), \quad s_{n}:=2 b^{1-n},
$$

where $a>1$ from $\left\{a_{k} ; k \geqslant 1\right\}$ and $b \in(1,2)$ to be determined below. Observe that $\Phi\left(r_{n}\right)=a \Phi\left(r_{n+1}\right)$. Clearly,

$$
\varphi_{a}\left(r_{n+1}\right)=r_{n} \text { and } r_{n} \downarrow 0, s_{n} \downarrow 0 \text {. }
$$

For simplicity of notation, we write

$$
Q_{n}:=Q\left(r_{n}\right), \quad M_{n}:=\sup _{Q_{n}} u, \quad m_{n}:=\inf _{Q_{n}} u .
$$


We are going to prove that the oscillation of $u$ over $Q_{k}$

$$
\operatorname{osc}_{Q_{k}} u:=M_{k}-m_{k} \leqslant s_{k}, \quad k \in \mathbb{N} \text {. }
$$

If this is proven, then (2.6) follows. In fact, for any $(t, x) \in Q_{1}$, there is an $n \in \mathbb{N}$ such that

$$
(t, x) \in Q_{n} \backslash Q_{n+1},
$$

which means that

$$
\Phi\left(r_{n+1}\right) \leqslant t<\Phi\left(r_{n}\right)=a \Phi\left(r_{n+1}\right) \text { or } r_{n+1} \leqslant|x|<r_{n} .
$$

In this case, we have

$$
|u(t, x)-u(0,0)| \leqslant M_{n}-m_{n} \leqslant s_{n}=2 b a^{-n \ln b / \ln a} \leqslant 2 b\left(\frac{t \vee \Phi(|x|)}{\Phi(r)}\right)^{\frac{\ln b}{\ln a}}
$$

and (2.6) follows with $\beta=\ln b / \ln a$.

(ii) We now prove (2.7) by an inductive argument. First of all, clearly,

$$
M_{1}-m_{1} \leqslant 1 \leqslant s_{1}=2, \quad M_{2}-m_{2} \leqslant 1 \leqslant s_{2}=2 / b .
$$

Next suppose that $M_{k}-m_{k} \leqslant s_{k}$ for all $k=1, \ldots, n$. Define

$$
A:=\left\{z \in D_{a}\left(r_{n+1}\right): u(z) \leqslant \frac{m_{n}+M_{n}}{2}\right\} .
$$

By considering $1-u$ instead of $u$ if necessary, we may assume that

$$
\mu_{r_{n+1}}(A) \geqslant \frac{1}{2} \mu_{r_{n+1}}\left(D_{a}\left(r_{n+1}\right)\right)
$$

where $\mu_{r_{n+1}}$ is given in $\left(\mathbf{H}_{2}\right)$. (Note here we are interested in the oscillation $\operatorname{osc}_{Q_{k}} u=$ $M_{k}-m_{k}$ not on the exact values of $M_{k}$ and $m_{k}$.) Since $\mu_{r_{n+1}}$ is regular, there is a compact subset $K \subset A$ such that

$$
\mu_{r_{n+1}}(K) \geqslant \frac{1}{3} \mu_{r_{n+1}}\left(D_{a}\left(r_{n+1}\right)\right) .
$$

For any $\varepsilon>0$, let us choose $z_{1}, z_{2} \in Q_{n+1}$ so that

$$
u\left(z_{1}\right) \leqslant m_{n+1}+\varepsilon, \quad u\left(z_{2}\right) \geqslant M_{n+1}-\varepsilon .
$$

If one can show

$$
u\left(z_{2}\right)-u\left(z_{1}\right) \leqslant s_{n+1},
$$


then

$$
M_{n+1}-m_{n+1}-2 \varepsilon \leqslant s_{n+1} \Rightarrow M_{n+1}-m_{n+1} \leqslant s_{n+1},
$$

and (2.7) is thus proven.

(iii) Now, we show (2.9). Since $z_{2} \in Q_{n+1} \subset Q_{n}$, if we define $\tau_{n}:=\tau_{Q_{n}}$, then by (2.2) we have

$$
\begin{aligned}
u\left(z_{2}\right)-u\left(z_{1}\right)= & \mathbb{E}_{z_{2}}\left[u\left(Z_{\tau_{n} \wedge \sigma_{K}}\right)-u\left(z_{1}\right)\right] \\
= & \left(\mathbb{E}_{z_{2}}\left[u\left(Z_{\sigma_{K}}\right)-u\left(z_{1}\right) ; \sigma_{K}<\tau_{n}\right]\right. \\
& \left.+\mathbb{E}_{z_{2}}\left[u\left(Z_{\tau_{n}}\right)-u\left(z_{1}\right) ; \sigma_{K} \geqslant \tau_{n} ; Z_{\tau_{n}} \in Q_{n-1}\right]\right) \\
& +\mathbb{E}_{z_{2}}\left[u\left(Z_{\tau_{n}}\right)-u\left(z_{1}\right) ; \sigma_{K} \geqslant \tau_{n}, Z_{\tau_{n}} \notin Q_{n-1}\right] \\
= & : I_{1}+I_{2} .
\end{aligned}
$$

For $I_{1}$, since $u\left(z_{1}\right) \geqslant m_{n+1} \geqslant m_{n} \geqslant m_{n-1}$, by the inductive hypothesis we have

$$
\begin{aligned}
I_{1} & \leqslant\left(\frac{m_{n}+M_{n}}{2}-m_{n}\right) \mathbb{P}_{z_{2}}\left(\sigma_{K}<\tau_{n}\right)+\left(M_{n-1}-m_{n-1}\right) \mathbb{P}_{z_{2}}\left(\sigma_{K} \geqslant \tau_{n}\right) \\
& \leqslant \frac{s_{n}}{2} \mathbb{P}_{z_{2}}\left(\sigma_{K}<\tau_{n}\right)+s_{n-1}\left(1-\mathbb{P}_{z_{2}}\left(\sigma_{K}<\tau_{n}\right)\right) \\
& \leqslant s_{n-1}\left(1-\mathbb{P}_{z_{2}}\left(\sigma_{K}<\tau_{n}\right) / 2\right) \leqslant s_{n+1} b^{2}\left(1-\gamma_{a} /(2 a)\right),
\end{aligned}
$$

where the last step is due to (2.8) and $\left(\mathbf{H}_{2}\right)$. For $I_{2}$, we similarly have

$$
\begin{aligned}
I_{2}= & \sum_{i=1}^{n-2} \mathbb{E}_{z_{2}}\left[u\left(Z_{\tau_{n}}\right)-u\left(z_{1}\right) ; \sigma_{K} \geqslant \tau_{n}, Z_{\tau_{n}} \in Q_{n-i-1} \backslash Q_{n-i}\right] \\
& +\mathbb{E}_{z_{2}}\left[u\left(Z_{\tau_{n}}\right)-u\left(z_{1}\right) ; \sigma_{K} \geqslant \tau_{n}, Z_{\tau_{n}} \notin Q_{1}\right] \\
\leqslant & \sum_{i=1}^{n-2} s_{n-i-1} \mathbb{P}_{z_{2}}\left(Z_{\tau_{n}} \notin Q_{n-i}\right)+\mathbb{P}_{z_{2}}\left(Z_{\tau_{n}} \notin Q_{1}\right) .
\end{aligned}
$$

Noticing that

$$
\mathbb{P}_{z_{2}}\left(Z_{\tau_{n}} \notin Q_{n-i}\right)=\mathbb{P}_{z_{2}}\left(X_{\left.\tau_{Q\left(r_{n}\right.}\right)} \notin B\left(r_{n-i}\right)\right)
$$

by $\left(\mathbf{H}_{1}\right)$, we further have for $a>\max \left\{C_{4}, b\right\}$,

$$
\begin{aligned}
I_{2} & \leqslant C_{3} \sum_{i=1}^{n-2} s_{n-i-1} \frac{\Phi\left(r_{n}\right)}{\Phi\left(r_{n-i}\right)}+C_{3} \frac{\Phi\left(r_{n}\right)}{\Phi\left(r_{1}\right)}=2 C_{3} b^{2-n} \sum_{i=1}^{n-2}(b / a)^{i}+C_{3} a^{1-n} \\
& \leqslant s_{n+1} b^{2}\left(\frac{C_{3} b}{a-b}+\frac{C_{3}}{2 a}\right)
\end{aligned}
$$


which together with (2.10) and (2.11), yields that

$$
u\left(z_{2}\right)-u\left(z_{1}\right) \leqslant s_{n+1} b^{2}\left(1-\frac{\gamma_{a}}{2 a}+\frac{C_{3} b}{a-b}+\frac{C_{3}}{2 a}\right) \leqslant s_{n+1} b^{2}\left(1-\frac{\gamma_{a}}{3 a}\right) \leqslant s_{n+1}
$$

provided we take $a=a_{k}$ large enough and $b$ close to 1 as $\lim _{k \rightarrow \infty} \gamma_{a_{k}}=\infty$. This completes the proof.

\section{Proof of Theorem 1.1}

We first recall the definition and properties of regularly varying functions.

Definition 3.1 A measurable and positive function $\phi:(0,2) \rightarrow(0, \infty)$ is said to vary regularly at zero with index $\alpha \in \mathbb{R}$ if for every $\lambda>0$,

$$
\lim _{r \rightarrow 0} \frac{\phi(\lambda r)}{\phi(r)}=\lambda^{\alpha}
$$

We call such $\phi$ a regularly varying function. All regularly varying functions with index $\alpha$ is denoted by $\mathscr{R}_{\alpha}$.

We list some properties of $\phi \in \mathscr{R}_{\alpha}$ for later use (cf. [3, pp. 25-28] and [11]).

Proposition 3.2 Let $\alpha \geqslant 0$ and $\phi \in \mathscr{R}_{\alpha}$ be bounded away from 0 and $\infty$ on any compact subset of $(0,2)$. For any $\delta>0$, there is a constant $C_{5}=C_{5}(\delta) \geqslant 1$ such that for all $r, s \in(0,1]$,

$$
\frac{\phi(r)}{\phi(s)} \leqslant C_{5} \max \left\{\left(\frac{r}{s}\right)^{\alpha+\delta},\left(\frac{r}{s}\right)^{\alpha-\delta}\right\}
$$

and for any $\beta>\alpha-1$,

$$
\begin{array}{r}
\lim _{r \rightarrow 0} \frac{\phi(r)}{r^{\beta+1}} \int_{0}^{r} \frac{s^{\beta}}{\phi(s)} \mathrm{d} s=(\beta-\alpha+1)^{-1}, \\
\lim _{r \rightarrow 0} r^{\beta+1-\alpha} \phi(r) \int_{r}^{2} \frac{1}{s^{\beta+2-\alpha} \phi(s)} \mathrm{d} s=(\beta-\alpha+1)^{-1} .
\end{array}
$$

Moreover, if we define

$$
\Phi(r):=\left(\int_{r}^{2} \frac{1}{\phi(s) s} \mathrm{~d} s\right)^{-1}
$$

then $\Phi \in \mathscr{R}_{\alpha}$ and

$$
\lim _{r \rightarrow 0} \frac{\Phi(r)}{\phi(r)}=\alpha
$$


In particular, (3.1) and (3.2) also hold for $\Phi$, and for some $C_{6}>1$,

$$
\phi(2 s) \leqslant C_{6} \phi(s) \text { and } \Phi(2 s) \leqslant C_{6} \Phi(s) \text { for } s \in(0,1 / 2) \text {. }
$$

We now return to the setting in Sect. 1. By normalizing the function $\phi$ in (1.3) by a constant multiple, we may and do assume the scale function $\Phi$ defined by (1.7) has the property that $\Phi(1)=1$. Consider the nonlocal operator $\mathscr{L}_{t}^{b}$ in (1.6). We assume (MP) The martingale problem for $\left(\mathscr{L}_{t}^{b}, C_{c}^{\infty}\left(\mathbb{R}^{+} \times \mathbb{R}^{d}\right)\right)$ is well posed for every initial value $(t, x) \in \mathbb{R}^{+} \times \mathbb{R}^{d}$.

Denote by $\mathbb{P}_{(t, x)}$ the law of the unique solution to the martingale problem for $\left(\mathscr{L}_{t}^{b}, C_{c}^{\infty}\left(\mathbb{R}^{+} \times \mathbb{R}^{d}\right)\right)$ with initial value $(t, x) \in \mathbb{R}^{+} \times \mathbb{R}^{d}$. By [10, Theorems 4.3 .12 and 4.4.2] $), Z_{s}=\left(V_{0}+s, X_{s}\right)$ is a Hunt process with $\mathbb{P}_{(t, x)}\left(V_{0}=t\right.$ and $\left.X_{0}=x\right)=1$ and so it has a Lévy system that describes the jumps of $Z$. By a similar argument as that for [4, Theorem 2.6], we have the following.

Theorem 3.3 Assume (MP) holds. Then for any $(t, x) \in \mathbb{R}^{+} \times \mathbb{R}^{d}$ and any nonnegative measurable function $f$ on $\mathbb{R}^{+} \times \mathbb{R}^{d} \times \mathbb{R}^{d}$ vanishing on $\left\{(s, x, y) \in \mathbb{R}^{+} \times \mathbb{R}^{d} \times \mathbb{R}^{d}\right.$ : $x=y\}$ and $\left(\mathscr{F}_{t}\right)$-stopping time $T$,

$$
\mathbb{E}_{(t, x)}\left[\sum_{s \leqslant T} f\left(s, X_{s-}, X_{s}\right)\right]=\mathbb{E}_{(t, x)}\left[\int_{0}^{T}\left(\int_{\mathbb{R}^{d}} f\left(s, X_{s}, y\right) \kappa_{s+t}\left(X_{s}, y-X_{s}\right) \mathrm{d} y\right) \mathrm{d} s\right] .
$$

Next we prove the following estimate, which implies $\left(\mathbf{H}_{1}\right)$.

Lemma 3.4 Let $C_{6}$ be as in (3.6). Under (1.2), (1.3) and (MP), there is a constant $C_{7} \geqslant 1$ such that for all $a \geqslant C_{6}$ and $r, R \in(0,1)$ with $\varphi_{a}(r) \leqslant R$,

$$
\sup _{\left(t_{0}, x_{0}\right) \in Q(r)} \mathbb{P}_{\left(t_{0}, x_{0}\right)}\left(X_{\tau_{Q(r)}} \notin B(R)\right) \leqslant C_{7} \frac{\Phi(r)}{\Phi(R)},
$$

where $\Phi$ is defined by (3.4).

Proof For simplicity of notation, we write $z=\left(t_{0}, x_{0}\right)$. Note that $r<\varphi_{a}(r)$ so we have by formula (3.7),

$$
\begin{aligned}
& \mathbb{P}_{z}\left(X_{\tau_{Q(r)}} \notin B(R)\right)=\mathbb{E}_{z}\left(\sum_{0<s \leqslant \tau_{Q(r)}} \mathbf{1}_{\left\{X_{s-} \in B(r), X_{s} \in B(R)^{c}\right\}}\right) \\
& =\mathbb{E}_{z} \int_{0}^{\tau_{Q(r)}} \int_{B(R)^{c}} \kappa_{s+t}\left(X_{s}, X_{s}-y\right) \mathrm{d} y \mathrm{~d} s \\
& =\mathbb{E}_{z} \int_{0}^{\tau_{Q(r)}} \int_{B(2) \cap B(R)^{c}} \kappa_{s+t}\left(X_{s}, X_{S}-y\right) \mathrm{d} y \mathrm{~d} s \\
& +\mathbb{E}_{z} \int_{0}^{\tau_{Q(r)}} \int_{B(2)^{c}} \kappa_{s+t}\left(X_{s}, X_{s}-y\right) \mathrm{d} y \mathrm{~d} s=: I_{1}+I_{2} .
\end{aligned}
$$


By (3.6), we have $\varphi_{a}(r) \geqslant 2 r$ for $a \geqslant C_{6}$, which implies that

$$
|x-y| \geqslant|y|-|x| \geqslant|y| / 2 \text { for } x \in B(r) \text { and } y \in B(R)^{c} \subset B\left(\varphi_{a}(r)\right)^{c} \text {. }
$$

For $I_{1}$, by (1.3) and (3.1) we have

$$
\begin{aligned}
I_{1} & \leqslant \mathbb{E}_{z} \int_{0}^{\tau_{Q(r)}} \int_{B(2) \cap B(R)^{c}} \frac{c_{1}^{-1}}{\phi\left(\left|X_{s}-y\right|\right)\left|X_{s}-y\right|^{d}} \mathrm{~d} y \mathrm{~d} s \\
& \leqslant C \mathbb{E}_{z} \tau_{Q(r)} \int_{B(2) \cap B(R)^{c}} \frac{\mathrm{d} y}{\phi(|y|)|y|^{d}} \leqslant C \mathbb{E}_{z} \tau_{Q(r)} / \Phi(R) .
\end{aligned}
$$

On the other hand, by (1.2) we clearly have

$$
I_{2} \leqslant \mathbb{E}_{z} \int_{0}^{\tau_{Q(r)}} \int_{B(1)^{c}} \kappa_{s+t}\left(X_{s}, y\right) \mathrm{d} y \mathrm{~d} s \leqslant C_{1} \mathbb{E}_{z} \tau_{Q(r)} .
$$

Hence, by (1.8),

$$
\mathbb{P}_{z}\left(X_{\tau_{Q(r)}} \notin B(R)\right) \leqslant \mathbb{E}_{z} \tau_{Q(r)}\left(C_{1}+C / \Phi(R)\right) \leqslant C_{7} \mathbb{E}_{z} \tau_{Q(r)} / \Phi(R),
$$

which yields the desired estimate by $\tau_{Q(r)} \leqslant \Phi(r)$.

Before verifying $\left(\mathbf{H}_{2}\right)$, we need the following lemma.

Lemma 3.5 Let $\Phi$ be defined by (3.4). Suppose that one of the following conditions holds:

(i) $\liminf _{r \rightarrow 0} r / \Phi(r)=0$ and for some $C_{2}>0$,

$$
\frac{\Phi(r)}{\Phi(s)} \leqslant C_{2} \frac{r}{s}, \quad 0<s \leqslant r \leqslant 1
$$

and for some $C_{b}>0$,

$$
\left|b_{t}(x)\right| \leqslant C_{b}|x| / \Phi(|x|), \quad|x| \leqslant 1 .
$$

(ii) $\liminf _{r \rightarrow 0} r / \Phi(r)>0$ and $b$ is bounded measurable.

Then there exists a constant $C_{8} \geqslant 1$ such that for all $r \in(0,1), x_{0} \in B(r)$ and $t_{0} \in[0,1]$,

$$
\mathbb{P}_{\left(t_{0}, x_{0}\right)}\left(\tau_{B\left(x_{0}, r\right)}<t\right) \leqslant \frac{C_{8} t}{\Phi(r)}, \quad t>0 .
$$

In particular, for any $a \geqslant C_{6}^{2}>1$ and $r \in(0,1)$ with $\varphi_{a}(r) \leqslant 1$,

$$
\sup _{x_{0} \in B(r)} \mathbb{P}_{\left(t_{0}, x_{0}\right)}\left(\tau_{Q\left(\varphi_{a}(r)\right)}<\Phi(r)\right) \leqslant \frac{C_{8}}{\sqrt{a}},
$$


where $C_{6}$ is the positive constant in (3.6).

Proof Given $f \in C_{b}^{2}\left(\mathbb{R}^{d}\right)$ with $f(0)=0$ and $f(x)=1$ for $|x| \geqslant 1$, set

$$
f_{r}(x):=f\left(\left(x-x_{0}\right) / r\right), \quad r>0 .
$$

By the optional stopping theorem,

$$
\mathbb{P}_{\left(t_{0}, x_{0}\right)}\left(\tau_{B\left(x_{0}, r\right)}<t\right) \leqslant \mathbb{E}_{\left(t_{0}, x_{0}\right)} f_{r}\left(X_{\tau_{B\left(x_{0}, r\right)} \wedge t}\right)=\mathbb{E}_{\left(t_{0}, x_{0}\right)} \int_{0}^{\tau_{B\left(x_{0}, r\right)} \wedge t} \mathscr{L}_{s+t_{0}}^{b} f_{r}\left(X_{S}\right) \mathrm{d} s .
$$

On the other hand, by the definition of $\mathscr{L}_{s}^{b}$ and (1.3), we have

$$
\begin{aligned}
\left|\mathscr{L}_{s}^{b} f_{r}(x)\right|= & \left|\int_{\mathbb{R}^{d}}\left(f_{r}(x+z)+f_{r}(x-z)-2 f_{r}(x)\right) \kappa_{s}(x, z) \mathrm{d} z+b_{s}(x) \cdot \nabla f_{r}(x)\right| \\
\leqslant & C \int_{|z| \leqslant r} \frac{\left\|\nabla^{2} f_{r}\right\|_{\infty}}{\phi(|z|)|z|^{d-2}} \mathrm{~d} z \\
& +C \int_{1 \geqslant|z| \geqslant r} \frac{\left\|f_{r}\right\|_{\infty}}{\phi(|z|)|z|^{d}} \mathrm{~d} z+\left\|f_{r}\right\|_{\infty} \int_{|z| \geqslant 1} \kappa_{s}(x, z) \mathrm{d} z+\left\|\nabla f_{r}\right\|_{\infty}\left|b_{s}(x)\right| \\
\leqslant & \frac{C}{r^{2}} \int_{0}^{r} \frac{s \mathrm{~d} s}{\phi(s)}+C \int_{r}^{1} \frac{\mathrm{d} s}{\phi(s) s}+C+\frac{C\left|b_{s}(x)\right|}{r} \\
\leqslant & \frac{C}{\phi(r)}+\frac{C}{\Phi(r)}+C+\frac{C\left|b_{s}(x)\right|}{r} \quad \text { by (3.2) and (3.3). }
\end{aligned}
$$

Substituting this into (3.12) and using (3.5) and (1.8), we obtain

$$
\mathbb{P}_{\left(t_{0}, x_{0}\right)}\left(\tau_{B\left(x_{0}, r\right)}<t\right) \leqslant \frac{C t}{\Phi(r)}+\mathbb{E}_{\left(t_{0}, x_{0}\right)} \int_{0}^{\tau_{B\left(x_{0}, r\right)} \wedge t} \frac{C\left|b_{s+t_{0}}\left(X_{s}\right)\right|}{r} \mathrm{~d} s
$$

In case (i), since

$$
|x| \leqslant\left|x-x_{0}\right|+\left|x_{0}\right| \leqslant 2 r \text { for } x \in B\left(x_{0}, r\right) \text { and } x_{0} \in B(r)
$$

(3.10) follows by (3.13) and

$$
\frac{\left|b_{s+t_{0}}(x)\right|}{r} \stackrel{(3.9)}{\leqslant} \frac{C_{b}|x|}{r \Phi(|x|)} \stackrel{(3.8)}{\leqslant} \frac{2 C_{b} C_{2}}{\Phi(2 r)} \leqslant \frac{C}{\Phi(r)} .
$$

In case (ii), (3.10) follows by (3.13) and $\left|b_{s+t_{0}}\left(X_{S}\right)\right| \leqslant\|b\|_{L^{\infty}\left(\mathbb{R}^{+} \times \mathbb{R}^{d}\right)}$ as well as $\frac{1}{r} \leqslant \frac{C}{\Phi(r)}$.

On the other hand, by (3.6), we have for any $a \geqslant C_{6}^{2}$,

$$
\Phi\left(2 \varphi_{\sqrt{a}}(r)\right) \leqslant C_{6} \sqrt{a} \Phi(r) \leqslant a \Phi(r),
$$


which implies that for $x_{0} \in B(r)$ and $x \in B\left(x_{0}, \varphi_{\sqrt{a}}(r)\right)$,

$$
|x| \leqslant\left|x-x_{0}\right|+\left|x_{0}\right| \leqslant \varphi_{\sqrt{a}}(r)+r \leqslant 2 \varphi_{\sqrt{a}}(r) \leqslant \varphi_{a}(r) .
$$

Hence $B\left(x_{0}, \varphi_{\sqrt{a}}(r)\right) \subset B\left(\varphi_{a}(r)\right)$ and

$$
\mathbb{P}_{\left(t_{0}, x_{0}\right)}\left(\tau_{Q\left(\varphi_{a}(r)\right)}<\Phi(r)\right) \leqslant \mathbb{P}_{\left(t_{0}, x_{0}\right)}\left(\tau_{B\left(x_{0}, \varphi_{\sqrt{a}}(r)\right)}<\Phi(r)\right) \stackrel{(3.10)}{\leqslant} \frac{C_{8} \Phi(r)}{\Phi\left(\varphi_{\sqrt{a}}(r)\right)}=\frac{C_{8}}{\sqrt{a}}
$$

The proof is complete.

For $a>1$ and $r \in(0,1)$ with $\varphi_{a}(r) \leqslant 1$, let $D_{a}(r)$ be defined by (2.3). Define a measure

$$
\mu_{r}(A):=\int_{0}^{\Phi(r)} \int_{\mathbb{R}^{d}} \frac{1_{A}(s, y) \Phi(|y|)}{\phi(|y|)|y|^{d}} \mathrm{~d} y \mathrm{~d} s, \quad A \subset D_{a}(r) .
$$

The above definition of $\mu_{r}$ arises naturally when estimating the lower bound of $\mathbb{P}_{\left(t_{0}, x_{0}\right)}\left(\sigma_{K}<\tau_{Q\left(\varphi_{a}(r)\right)}\right)$ using the Léve system of $Z$; see (3.16) below. Clearly, we have

$$
\begin{aligned}
\mu_{r}\left(D_{a}(r)\right) & =\Phi(r) \int_{\varphi_{\sqrt{a}}(r) \leqslant|y| \leqslant \varphi_{a}(r)} \frac{\Phi(|y|)}{\phi(|y|)|y|^{d}} \mathrm{~d} y=\omega_{d} \Phi(r) \int_{\varphi_{\sqrt{a}}(r)}^{\varphi_{a}(r)} \frac{\Phi(s)}{\phi(s) s} \mathrm{~d} s \\
& =\omega_{d} \Phi(r) \int_{\varphi_{\sqrt{a}}(r)}^{\varphi_{a}(r)} \frac{1}{\Phi(s)} d \Phi(s)=\frac{1}{2} \omega_{d} \Phi(r) \ln a
\end{aligned}
$$

where $\omega_{d}$ is the sphere area of the unit ball.

Lemma 3.6 Suppose (1.2), (1.3), (MP) and the assumptions of Lemma 3.5 hold. There exist $a_{0} \geqslant 1$ and $c_{2} \in(0,1)$ such that for each $a \geqslant a_{0}$ and $r \in(0,1)$ with $\varphi_{a}(r) \leqslant 1$, and any compact subset $K \subset D_{a}(r)$ with $\mu_{r}(K)>\frac{1}{3} \mu_{r}\left(D_{a}(r)\right)$,

$$
\inf _{\left(t_{0}, x_{0}\right) \in Q(r)} \mathbb{P}_{\left(t_{0}, x_{0}\right)}\left(\sigma_{K}<\tau_{Q\left(\varphi_{a}(r)\right)}\right) \geqslant c_{2} \frac{\ln a}{a} .
$$

In particular, condition $\left(\mathbf{H}_{2}\right)$ holds.

Proof Notice that

$$
\left\{Z_{\tau_{Q\left(\varphi_{\sqrt{a}}(r)\right)}} \in K\right\} \subset\left\{\sigma_{K}<\tau_{Q\left(\varphi_{a}(r)\right)}\right\}
$$

It suffices to prove that there are $a_{0} \geqslant 1$ and $c_{2} \in(0,1)$ such that for all $a \geqslant a_{0}$ and any $\left(t_{0}, x_{0}\right) \in Q(r)$,

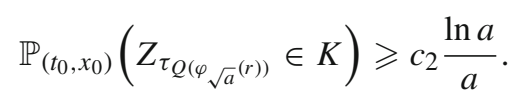


As $\mu_{r}\left(\partial Q\left(\varphi_{\sqrt{a}}(r)\right)\right)=0$, by taking a suitable subset of $K$ if needed, we may assume without loss of generality that $\left.K \cap \partial Q\left(\varphi_{\sqrt{a}}(r)\right)\right)=\emptyset$. Then

$$
\mathbf{1}_{K}\left(Z_{\left.\tau_{Q(\varphi \sqrt{a}}(r)\right)}\right)=\sum_{\left.0<s \leqslant \tau_{Q(\varphi \sqrt{a}}(r)\right)} \mathbf{1}_{X_{s-} \neq X_{s}} \mathbf{1}_{K}\left(Z_{S}\right) .
$$

Hence by formula (3.7) and (1.3), we have

$$
\begin{aligned}
\mathbb{P}_{\left(t_{0}, x_{0}\right)}\left(Z_{\left.\tau_{Q(\varphi \sqrt{a}}(r)\right)} \in K\right) & =\mathbb{E}_{\left(t_{0}, x_{0}\right)} \int_{0}^{\tau_{Q\left(\varphi_{a}\right.}^{(r))}} \int_{\mathbb{R}^{d}} \mathbf{1}_{K}(s, y) \kappa_{S}\left(X_{s}, X_{s}-y\right) \mathrm{d} y \mathrm{~d} s \\
& \geqslant c_{1} \mathbb{E}_{\left(t_{0}, x_{0}\right)} \int_{0}^{\tau_{Q(\varphi} \sqrt{a}^{(r))}} \int_{\mathbb{R}^{d}} \frac{\mathbf{1}_{K}(s, y)}{\phi\left(\left|X_{s}-y\right|\right)\left|X_{s}-y\right|^{d}} \mathrm{~d} y \mathrm{~d} s .
\end{aligned}
$$

Since $|x-y| \leqslant 2|y|$ for $x \in B\left(\varphi_{\sqrt{a}}(r)\right)$ and $y \notin B\left(\varphi_{\sqrt{a}}(r)\right)$, by (3.1) and definition of $\mu_{r}$, we have

$$
\begin{aligned}
\mathbb{P}_{\left(t_{0}, x_{0}\right)}\left(Z_{\left.\tau_{Q(\varphi, \sqrt{a}}(r)\right)} \in K\right) & \geqslant c_{3} \mathbb{E}_{\left(t_{0}, x_{0}\right)}\left(\int_{0}^{\left.\tau_{Q(\varphi \sqrt{a}}(r)\right)} \int_{\mathbb{R}^{d}} \frac{\mathbf{1}_{K}(s, y)}{\phi(|y|)|y|^{d}} \mathrm{~d} y \mathrm{~d} s\right) \\
& =c_{3} \mathbb{E}_{\left(t_{0}, x_{0}\right)}\left(\int_{0}^{\left.\tau_{Q(\varphi}{ }^{(}(r)\right)} \int_{\mathbb{R}^{d}} \frac{\mathbf{1}_{K}(s, y)}{\Phi(|y|)} \mu_{r}(\mathrm{~d} y, \mathrm{~d} s)\right) \\
& \geqslant \frac{c_{3} \mu_{r}(K)}{\Phi\left(\varphi_{a}(r)\right)} \mathbb{P}_{\left(t_{0}, x_{0}\right)}\left(\tau_{Q\left(\varphi_{\sqrt{a}}(r)\right)} \geqslant \Phi(r)\right),
\end{aligned}
$$

where the last inequality is due to $y \in D_{a}(r)$ and the increasing of $\Phi$. Lastly, by $\mu_{r}(K) \geqslant \frac{1}{3} \mu_{r}\left(D_{a}(r)\right),(3.14)$ and (3.11), we obtain that for $a \geqslant C_{6}^{2} \vee 4 C_{8}^{2}=: a_{0}$,

$$
\begin{aligned}
\mathbb{P}_{\left(t_{0}, x_{0}\right)}\left(Z_{\left.\tau_{Q\left(\varphi_{\sqrt{a}}(r)\right)} \in K\right)} \geqslant \frac{c_{3} \omega_{d} \ln a}{6 a}\left(1-\mathbb{P}_{\left(t_{0}, x_{0}\right)}\left(\tau_{Q\left(\varphi_{\sqrt{a}}(r)\right)}<\Phi(r)\right)\right)\right. \\
\geqslant \frac{c_{3} \omega_{d} \ln a}{6 a}\left(1-\frac{C_{8}}{\sqrt{a}}\right) \geqslant \frac{c_{3} \omega_{d} \ln a}{12 a} .
\end{aligned}
$$

The proof is completed by taking $c_{2}=\frac{c_{3} \omega_{d}}{12}$.

We can now present the

Proof of Theorem 1.1 Fix $t_{0}>0$ and $x_{0} \in \mathbb{R}^{d}$.

(i) In this case, by assumption $b_{t}(x)$ is continuous in $x$ and $b_{t}(x) \leqslant C(1+|x|)$ for all $t>0$ and $x \in \mathbb{R}^{d}$. Thus, by the theory of ODE, the following ODE admits at least one solution $y_{t}$ for $t \in\left[0, t_{0}\right]$ :

$$
\dot{y}_{t}=-b_{t_{0}-t}\left(x_{0}+y_{t}\right), \quad y_{0}=0 \text {. }
$$

Define

$$
w(t, x):=u\left(t_{0}, x_{0}\right)-u\left(t_{0}-t, x_{0}+x+y_{t}\right)
$$


and

$$
\tilde{b}_{t}(x):=b_{t}\left(x+x_{0}+y_{t}\right)-b_{t}\left(x_{0}+y_{t}\right) .
$$

Then

$$
\partial_{t} w+\mathscr{L}_{t_{0}-t}^{\tilde{b}} w=0, \quad t \in\left[0, t_{0}\right) .
$$

Notice that by (1.11),

$$
\left|\tilde{b}_{t}(x)\right| \leqslant C_{b}|x| / \Phi(|x|) \text { for }|x| \leqslant 1 .
$$

By Lemmas 3.4-3.6 and Theorem 2.4, we have

$$
\begin{aligned}
|w(t, x)| & =|w(t, x)-w(0,0)| \\
& \leqslant 8\left(\frac{t \vee \Phi(|x|)}{t_{0}}\right)^{\beta}\|w\|_{L^{\infty}\left(\left[0, t_{0}\right] \times \mathbb{R}^{d}\right)} \quad \text { for }(t, x) \in Q\left(\Phi^{-1}\left(t_{0}\right)\right) .
\end{aligned}
$$

By making the change of variables $t_{0}-t=t^{\prime}$ and $x_{0}+x+y_{t}=x^{\prime}$, and noticing that

$$
\left|y_{t}\right| \leqslant \lambda t \text { for some } \lambda=\lambda\left(\|b /(1+|x|)\|_{\infty}\right)>0 \text {, }
$$

we obtain the desired estimate (1.12).

(ii) In this case, define

$$
w(t, x):=u\left(t_{0}, x_{0}\right)-u\left(t_{0}-t, x_{0}+x\right) .
$$

Just as above, one can conclude that (1.13) holds.

Acknowledgments The research of ZC was partially supported by NSF Grant DMS-1206276. The research of XZ was partially supported by NSFC Grant of China (Nos. 11271294, 11325105).

\section{References}

1. Abels, H., Kassmann, M.: The Cauchy problem and the martingale problem for integro-differential operators with non-smooth kernels. Osaka J. Math. 46, 661-683 (2009)

2. Bass, R.F., Levin, D.L.: Harnack inequalities for jump processes. Potential Anal. 17, 375-388 (2002)

3. Bingham, N.H., Goldie, C.M., Teugels, J.L.: Regular Variation. Cambridge University Press, Cambridge (1987)

4. Chen, Z.-Q., Kim, P., Song, R.: Dirichlet heat kernel estimates for fractional Laplacian with gradient perturbation. Ann. Probab. 40, 2483-2538 (2012)

5. Chen, Z.-Q., Kumagai, T.: Heat kernel estimates for stable-like processes on $d$-sets. Stoch. Process Appl. 108, 27-62 (2003)

6. Chen, Z.-Q., Kumagai, T.: Heat kernel estimates for jump processes of mixed types on metric measure spaces. Probab. Theory Relat. Fields 140, 277-317 (2008)

7. Chen, Z.-Q., Wang, L.: Uniqueness of stable processes with drift. arXiv:1309.6414

8. Chen, Z.-Q., Zhang, X.: Uniqueness of stable-like processes. In preparation 
9. Epstein, C.L., Pop, C.A.: Regularity for the supercritical fractional Laplacian with drift. arXiv:1309.5892v2 [math.AP]

10. Ethier, S.N., Kurtz, T.G.: Markov Processes: Characterization and Convergence. Wiley \& Sons, New York (1986)

11. Kassmann, M., Mimica, A.: Intrinsic scaling properties for nonlocal operators. arXiv:1310.5371v2

12. Krylov, N.V., Safonov, M.V.: An estimate for the probability of a diffusion process hitting a set of positive measure. Dokl. Akad. Nauk SSSR 245, 18-20 (1979)

13. Silvestre, L.: Hölder estimates for advection fractional-diffusion equations. Annali della Scuola Normale Superiore di Pisa Classe di Scienze 11(4), 843-855 (2012)

14. Zhang, $\mathrm{X} .: L^{p}$-maximal regularity of nonlocal parabolic equation and applications. Annales de $1^{\prime}$ Institut Henri Poincare Analyse non lineaire 30, 573-614 (2013) 